\title{
Controle da hemoterapia e da doença de Chagas transfusional: 1988 e 1990 *
}

\author{
Inquiry into the control of hemotherapy and transfusional Chagas' disease: 1988 \\ and 1990
}

\author{
Dalva Marli Valério Wanderley **, Thelma T. Gonzales ***, Maria Silvia C.A. Pereira **, \\ Rodrigo Delfino Nascimento ${ }^{* \star * *}$, Helio Moraes-Souza ${ }^{* * * *}$
}

\begin{abstract}
WANDERLEY, D.M.V. et al. Controle da hemoterapia e da doença de Chagas transfusional: 1988 e 1990. Rev. Saúde Pública, 27: 430-5, 1993. Com o objetivo de atualizar o estudo da situação da hemoterapia no Estado de São Paulo, Brasil foi realizado inquérito em 1990, comparando-o com outro realizado em 1988. Foram pesquisados 79 municípios, sendo que $(77,4 \%)$ realizavam transfusōes de sangue, envolvendo 104 serviços, dos quais $13,5 \%$ se utilizavam de sangue proveniente de Hemocentros. Em 1988, o inquérito envolveu 57 municípios, 40 com atividade hemoterápica e 71 serviços, sendo que em $8,5 \%$ foi constatada a participação de Hemocentros. $\mathrm{O}$ número de doadores, transfusōes e prevalência de sorologia positiva para a doença de Chagas foi de, respectivamente: $105.170,79.544$ e 1,24\%, enquanto que em 1988 estes números foram: 51.614, 49.211 e $1,52 \%$. Avaliando a triagem sorologica evidenciaram-se $94,2 \% ; 94,2 \% ; 94,2 \% ; 94,3 \%$ e $20,2 \%$ dos serviços que realizavam, respectivamente, testes sorológicos para doença de Chagas, sífilis, hepatite, HIV e malária, enquanto que em $1988,69,0 \% ; 70,4 \% ; 67,6 \% ; 63,4 \%$ e $16,9 \%$ dos serviços empregavam tais testes, respectivamente. Em relação à triagem sorológica específica para doença de Chagas, observou-se que: a reação de fixação do complemento era realizada por 4,9\% dos scrviços; látex por 4,0\%, imunofluorescência indireta 80\%; hemaglutinação direta 24\%; hemaglutinação indireta $69 \%$ e ELISA $59,0 \%$. No inquérito precedente apenas $14,3 \%$ dos serviços rcalizavam teste ELISA, e a fixação do complemento era utilizada em $28,6 \%$ dos serviços. Os resultados mostram que os serviços estão utilizando, a cada đia, técnicas mais sensíveis na rotina sorológica. Foi observado ainda que em $1988,77,6 \%$ de todos os serviços estudados realizavam apenas uma técnica para o diagnóstico da doença de Chagas, enquanto que em 1990, 92,9\% empregavam duas ou mais diferentes técnicas. Conclui-se que a qualidade da hemoterapia praticada no Estado de São Paulo foi significativamente melhorada nos dois últimos anos.
\end{abstract}

Descritores: Banco de sangue, estatística. Tripanossomose sul-americana, transmissão. Sorodiagnóstico, estatística.

\section{Introduçăo}

Várias são as doenças passívcis de transmissão através do sangue, destacando-sc a docnça de Chagas, a sífilis, a síndrome da imunodeficiência adquirida, a hepatite e a malária, entre outras. No que diz respcito

\footnotetext{
* Trabalho apresentado na VIII Reunião Anual de Pesquisa Aplicada em Doença de Chagas, Ubcraba, 1992.

** Superintendência de Controle de Endemias (SUCEN) São Paulo, SP - Brasil

*** Centro de Vigilância Sanitária (CVS) da Secretaria de Estado da Saúde de São Paulo - São Paulo, SP - Brasil Faculdade de Medicina do Triângulo Mineiro - Uberaba, MG - Brasil
}

Separatas/Reprints: D.M.V. Wanderley - Rua Paula Souza, 166 - 01027-000 - São Paulo, SP - Brasil

Edição subvencionada pela FAPESP. Processo Medicina 93/ 0208-5. à doença de Chagas, a hipótese da sua ocorrência, via transfusão de sangue, foi aventada pela primeira vez por Mazza ${ }^{3}$, cm 1936, na Argentina. Em 1949, Pellegrino ${ }^{5}$ realizou o primciro inquérito sorológico entre doadores e candidatos a doação de sangue de Belo Horizonte, encontrando uma prevalência de 1,7\%. Desde então vários inquéritos foram realizados em Bancos de Sangue, daquela cidade, encontrando-se percentuais entre 2,9 e $16,9 \%^{2}$. Ampla revisão realizada recentemente por Schimunis ${ }^{6}$, refere-se aos vários inquéritos realizados em Bancos de Sangue de todo o continente americano na última década, demonstrando prevalências que variam entre zero e $63 \%$, com uma média próxima de $3 \%$. Estes dados tornam-se bastante importantes quando se consideram estudos que mostram que $60 \%$ dos chagásicos crônicos apresentam Trypanossoma cruzi circulando $\mathrm{cm}$ seu sangue, e que o risco de aquisição de infecção chagásica através de um doador infectado é de 12,5 a $25 \%$ para uma única transfusão?. 
A primeira definição de uma política nacional na área de sangue e hemoderivados deu-se com a criação, em 1980, pelo Ministério da Saúde, da Pró-Sangue (hoje Coordenação de Sangue e Hemoderivados - $\operatorname{COSAH}$ ), dando início à criação dos Hemocentros estaduais. Entretanto, foi a partir da $8^{2}$ Conferência Nacional de Saúde, em 1986, com a premissa de que "é dever do Estado prover os meios para um atendimento hematológico e hemoterápico de acesso universal e de boa qualidade", que tal política foi melhor defini$\mathrm{da}^{2}$. Todavia, os dispositivos legais acerca da mesma tornaram-se públicos apenas em 1988, através da Lei 7.649 e decreto 95.721. No Estado de São Paulo, o decreto número 12.479 de 1978 determina o cadastramento de doadores com dados de identificação, exame clínico e resultado de provas laboratoriais, especificando para o caso da triagem da doença de Chagas c hepatite $\mathrm{B}$ a obrigatoriedade de realização, para cada caso, de no mínimo duas reações sorológicas adcquadas e difcrentes. Mais recentemente, a portaria $n^{9} 721$ / GM, de 9 de agosto de 1989, do Ministério da Saúde, determina, entre outros procedimentos, a obrigatoriedade da realização de sorologia para: sífilis, doença de.Chagas (por duas diferentes técnicas), hepatite B e HIV.

Com vistas a se obter uma "radiografia" da hemoterapia praticada no Brasil, com destaque para a doença de Chagas transfusional, no período de 1989/90, foi realizado um Inquérito Nacional onde foram investigados 850 municípios, scndo que $\mathrm{cm}$ $585(68,8 \%)$ sc constatou atividade hcmotcrápica. Dos 1.525 scrviços estudados, naqueles $585 \mathrm{mu}$ nicípios, apenas $66,9 \%$ declararam realizar tria$\mathrm{gcm}$ sorológica para Chagas. A prevalência de sorologia positiva entre os doadores foi de $0,97 \% \%^{4}$.

No citado inquérito, o Estado de São Paulo contribuiu com a avaliação de 57 municípios dos quais $40(70,2 \%)$ referiam prática hemoterápica, $\mathrm{e}$ dos 71 serviços pesquisados, $69 \%$ declararam realizar triagem sorologica para doença de Chagas, cuja prevalência de positividade entre os doadores foi de $1,5 \%$. Este quadro foi atualizado no ano de 1990 e seus resultados juntamente com aqueles obtidos no levantamento anterior, são apresentados no presente trabalho.

\section{Material e Método}

Para cada uma das 5 Macro-Regiócs de Saúde do Estado de São Paulo foram sorteados, através de amostra casual simples, 10 municípios, na seguinte ordem: 3 com até 10.000 habitantes, 4 com 10.001 a 50.000 e 3 com mais de 50.000 , alćm da scde da Macro-Região. Desta forma procurou-se estratificar melhor a amostra com base na densidade demográfica e na situação geográfica de cada região. Em cada município sorteado aplicava-se um questionário (formulário 1) para se obter informações sobre: população do municipio, existência de hospitais e de prática hemoterápica. Quando se constatava a realização de hemoterapia era aplicado um novo questionário (formulário 2), com os seguintes quesitos: 1) procedência do sangue; 2) categoria dos doadores; 3) utilização de sangue e componentes; 4) número de transfusões, número de doadores e número de soros positivos para $T$. cruzi; 5) exames utilizados para triagem dos doadores; 6) número e tipo de técnicas empregadas para triagem sorológica da doença de Chagas; 7) experiência prévia do serviço com o emprego da violeta de genciana na quimioprofilaxia da doença de Chagas.

Participaram do primeiro inquérito $57 \mathrm{mu}$ nicípios que responderam aos formulários utilizando-se de informaçõcs referentes às atividades desenvolvidas no ano de 1988, e do segundo, $62 \mathrm{com}$ dados do ano de 1990. Para o envio, preenchimento e devolução dos formulários contou-se com a participação dos responsáveis pela Vigilância Sanitária de cada município.

Um inquérito de consistência em $10 \%$ dos municípios sorteados foi realizado com o intuito de confirmar o processo de controle de qualidade dos resultados.

\section{Resultados}

Os 57 e 62 municípios inventariados nos anos de 1988 e 1990 corresponderam a, respectivamente, 9,2 c $13,0 \%$ da população existente e a $10,0 \%$ e $10,7 \%$ do total de municípios do Estado, tendo sido constatada a atividade hemoterápica respectivamente, em 70,2 e $77,4 \%$ dos mesmos (Tabela 1). Sua localização no Estado de São Pau-

Tabela 1. Controle da hemoterapia no Estado de São Paulo: 1988 e 1990. População, municipios e atividade hemoterápica.

\begin{tabular}{lcc}
\hline Abrangéncia & 1988 & 1990 \\
\hline População existente & 32.361 .700 & 33.855 .529 \\
População trabalhada & 2.973 .148 & 4.429 .435 \\
$\%$ & 9,2 & 13,1 \\
& 572 & 581 \\
Municipios existentes & 57 & 62 \\
Municípios trabalhados & 10,0 & 10,7 \\
$\%$ & 40 & 48 \\
Atividade hemoterápica & 70,2 & 77,4 \\
\% Municípios trabalhados & 7,0 & 8,3 \\
$\%$ Total de municipios & & \\
\hline
\end{tabular}


lo pode ser avaliada na Figura. Tabulando-se por tamanho de população (Tabcla 2) observou-se que a prática da atividade hemoterápica é mais comum nos municípios maiores, sendo discreta $(25,0 \%$ e $37,5 \%$, respectivamente em 1988 e 1990) nos municípios com menos de 10.000 habitantes.

Indicações sobre a qualidade da hcmoterapia praticada pelos diversos serviços pesquisados acham-se sumarizadas nas Tabelas 3 a 7 . Observamos que: a) $13,6 \%$ dos serviços utilizaram sangue proveniente do Sistema Hemocentro e 47,0\% eram de bancos de sangue externos em 1990, enquanto que em 1988, a rede oficial de hemocentros era responsável por $8,5 \%$ do sangue utilizado, observando-se também, nesse ano, percentual menor de serviços que se utilizavam de sangue proveniente de bancos de sangue externos $(32,4 \%)$. Não houve alteração na participação de serviços que se serviam de sangue de seus próprios bancos de sangue (Tabela 3); b) os 104 serviços pesquisados em 1990 realizaram 105.170 transfusões, 79.544 coletas de sangue, das quais 74.930 foram analisadas quanto à presença de $T$. cruzi, obtendo-se 987 resultados positivos, significando $1,3 \%$ de positividade, enquanto que os 71 serviços pesquisados em 1988 realizaram 51.614 transfusões de sangue provenientes de 49.211 coletas. A triagem sorológica para doença de Chagas em 33.956 doadores resultou em $751 \mathrm{com}$ sorologia positiva $(2,2 \%)$ (Tabela 4$) ; c)$ a triagem dos doadores para:

Tabela 2. Controle da hemoterapia no Estado de São Paulo: 1988 e 1990. Realização de atividade hemoterápica segundo tamanho do municipio.

\begin{tabular}{|c|c|c|c|c|c|c|}
\hline \multirow{4}{*}{$\begin{array}{c}\text { Tamanho do } \\
\text { município } \\
\text { (n de habitantes) }\end{array}$} & \multicolumn{3}{|c|}{1988} & \multicolumn{3}{|c|}{1990} \\
\hline & \multicolumn{3}{|c|}{ Municipios } & \multicolumn{3}{|c|}{ Municipios } \\
\hline & \multirow[t]{2}{*}{ Pesquisados } & \multicolumn{2}{|c|}{$\mathrm{C} /$ ativ. hemot. } & \multirow[t]{2}{*}{ Pesquisados } & \multicolumn{2}{|c|}{$\mathrm{C} /$ ativ. hemot } \\
\hline & & № & $\%$ & & $\mathrm{~N}^{2}$ & $\%$ \\
\hline $\begin{array}{c}<10.000 \\
10.001 \text { a } 50.000 \\
>50.000\end{array}$ & $\begin{array}{r}8 \\
33 \\
16\end{array}$ & $\begin{array}{r}2 \\
26 \\
12\end{array}$ & $\begin{array}{l}25,0 \\
78,7 \\
75,0\end{array}$ & $\begin{array}{r}8 \\
29 \\
25\end{array}$ & $\begin{array}{r}3 \\
25 \\
20\end{array}$ & $\begin{array}{l}37,5 \\
86,2 \\
80,0\end{array}$ \\
\hline Total & 57 & 40 & 70,2 & 62 & 48 & 77,4 \\
\hline
\end{tabular}

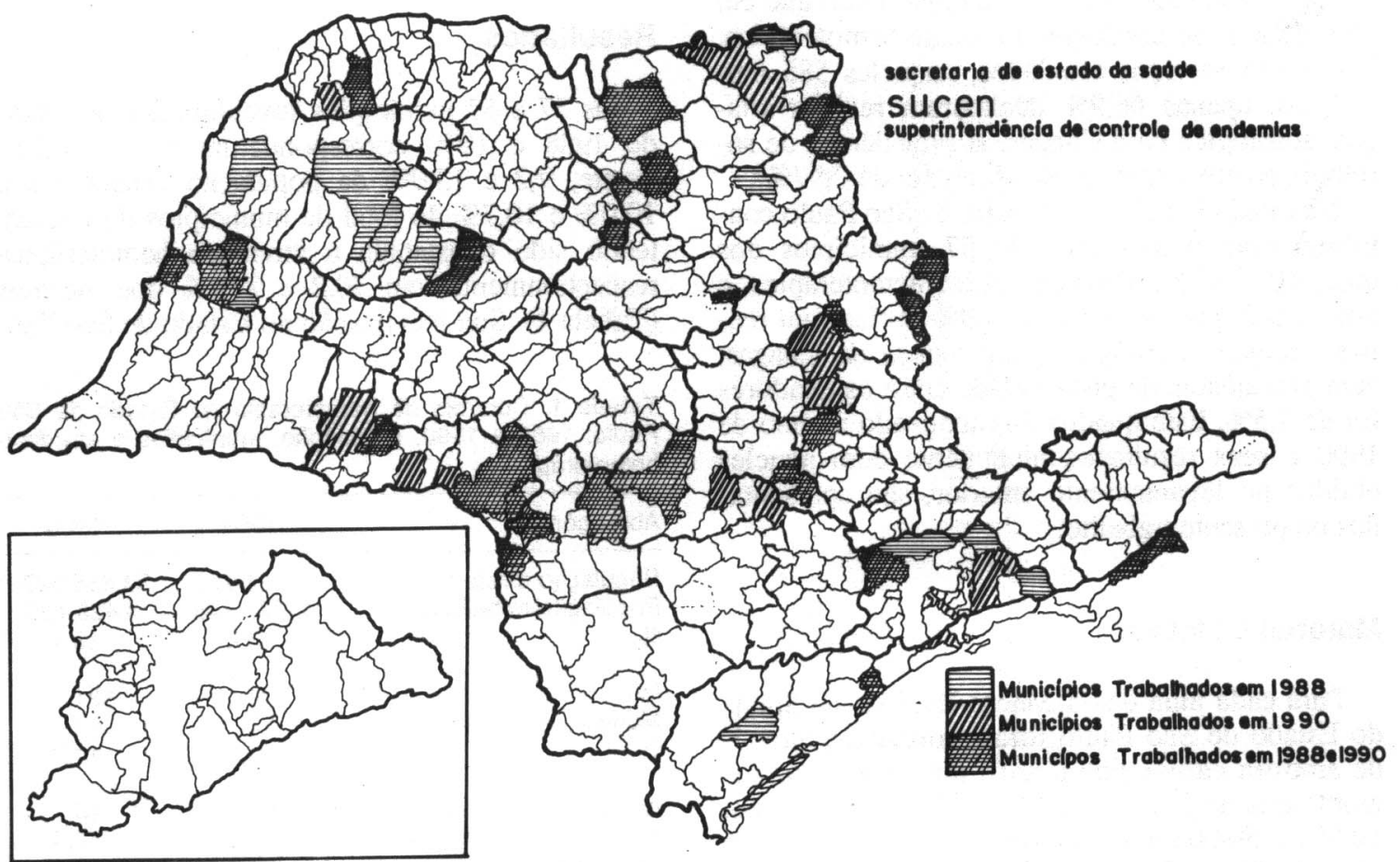

Figura. Controle da hemoterapia e da doença de Chagas transfusional no Estado de São Paulo, 1988 e 1990. 
Tabela 3. Controle da hemoterapia no Estado de São Paulo: 1988 e 1990. Procedência do sangue utilizado para transfusão.

\begin{tabular}{lcccccc}
\hline \multirow{2}{*}{ Procedência } & \multicolumn{2}{c}{1988} & & \multicolumn{2}{c}{1990} \\
\cline { 2 - 3 } \cline { 6 - 7 } & $\begin{array}{c}\text { Serviços } \\
\text { Hemoterápicos }\end{array}$ & & \multicolumn{2}{c}{$\begin{array}{c}\text { Serviços } \\
\text { Hemoterápicos }\end{array}$} \\
\cline { 2 - 3 } \cline { 6 - 7 } & $N^{2}$ & $\%$ & & Ne & $\%$ \\
\hline $\begin{array}{l}\text { Hemocentro } \\
\text { Banco de sangue }\end{array}$ & 6 & 8,5 & & 14 & 13,6 \\
externo & 23 & 32,4 & & 49 & 47,0 \\
$\begin{array}{l}\text { Banco de sangue } \\
\text { próprio }\end{array}$ & 28 & 39,4 & & 41 & 39,4 \\
Não resposta & 14 & 19,7 & & - & - \\
\hline Total & 71 & 100,0 & & 104 & 100,0 \\
\hline
\end{tabular}

Tabela 4. Controle da hemoterapia no Estado de São Paulo: 1988 e 1990. Número de transfusões, de doadores total e triados e com sorologia positiva para doença de Chagas.

\begin{tabular}{lcc}
\hline Condição & 1988 & 1990 \\
\hline Tranfusões & 51.614 & 105.170 \\
Doadores & 49.211 & 79.544 \\
Doadores com triagem & 33.956 & 74.930 \\
Doadores com sorologia positiva & 751 & 987 \\
$\%$ & 2,2 & 1,3 \\
\hline
\end{tabular}

Tabela 5. Controle da hemoterapia no Estado de São Paulo: 1988 e 1990. Serviços que fazem triagem sorológica dos doadores.

\begin{tabular}{lccccc}
\hline \multirow{2}{*}{ Triagem } & \multicolumn{2}{c}{1988} & & \multicolumn{2}{c}{1990} \\
\cline { 2 - 3 } \cline { 5 - 6 } & \multicolumn{2}{c}{$\begin{array}{c}\text { Serviços } \\
\text { Hemoterápicos }\end{array}$} & & \multicolumn{2}{c}{$\begin{array}{c}\text { Serviços } \\
\text { Hemoterápicos }\end{array}$} \\
\cline { 2 - 3 } \cline { 5 - 6 } & $N^{2}$ & $\% *$ & & $N^{2}$ & $\%^{* *}$ \\
\hline Chagas & 49 & 69,0 & & 98 & 94,2 \\
Sítilis & 50 & 70,4 & & 98 & 94,2 \\
Hepatite & 48 & 67,6 & & 98 & 94,2 \\
HIV & 45 & 63,4 & & 96 & 92,3 \\
Malária & 12 & 16,9 & & 21 & 20,2 \\
\hline
\end{tabular}

- Calculado sobre 71 serviços pesquisados.

* Calculado sobre 104 serviços pesquisados.

Chagas, Sífilis, Hepatite, HIV c Malária era feita, respectivamente, por $69 \%, 70,4 \%, 67,6 \% ; 63,4 \%$ e $16,9 \%$ dos Serviços em 1988, enquanto que em 1990 esses percentuais se clevaram para 94,2 , $94,2,94,2,92,3$ e 20,2 (Tabcla 5); d) entre as técnicas empregadas para triagem sorológica para doença de Chagas, as reaçðes de imunofluorescência indireta, aglutinação indireta, Gucrreiro Machado e ELISA foram realizadas respectivamente por $85,7,55,1,28,6$ e $14,3 \%$ dos serviços em 1988 e por $81,6,70,4,3,1$ e $60,2 \%$ dos serviços em
Tabela 6. Controle da hemoterapia no Estado de São Paulo: 1988 e 1990. Serviços segundo os exames utilizados na triagem sorológica da doença de Chagas.

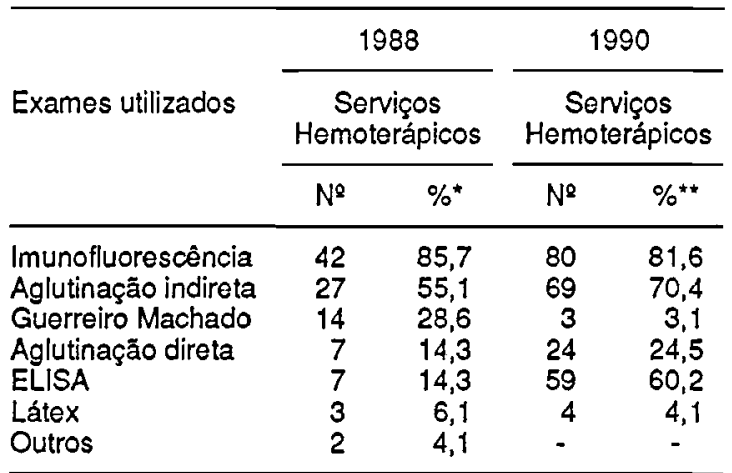

- Calculado sobre 49 serviços que fazem triagem sorológica para doença de Chagas.

* Calculado sobre 98 serviços que fazem triagem sorológica para doença de Chagas.

Tabela 7. Controle da hemoterapia no Estado de São Paulo: 1988 e 1990. Número de testes utilizados para triagem sorológica da doença de Chagas.

\begin{tabular}{lccccc}
\hline \multirow{2}{*}{$\begin{array}{l}\text { Número de } \\
\text { testes }\end{array}$} & \multicolumn{2}{c}{1988} & & \multicolumn{2}{c}{1990} \\
\cline { 2 - 3 } \cline { 5 - 6 } & \multicolumn{2}{c}{$\begin{array}{c}\text { Serviços } \\
\text { Hemoterápicos }\end{array}$} & & \multicolumn{2}{c}{$\begin{array}{c}\text { Serviços } \\
\text { Hemoterápicos }\end{array}$} \\
\cline { 2 - 3 } \cline { 5 - 6 } & $N^{2}$ & $\%$ & & $N^{2}$ & $\%$ \\
\hline 1 & 38 & 77,6 & & 7 & 7,1 \\
2 & 8 & 16,3 & & 57 & 58,2 \\
Mais que 2 & 3 & 6,1 & & 34 & 34,7 \\
\hline Total & 49 & 100,0 & & 98 & 100,0 \\
\hline
\end{tabular}

1990 (Tabela 6); e) entre os serviços que fizeram triagem sorológica para doença de Chagas em $1988,77,6 \%$ realizaram um único teste, 16,3 afirmaram fazcr 2 testcs e $6,1 \%$ mais do que 2 testes, enquanto que $\mathrm{cm} 1990$ esses percentuais foram de $7,1,59,2$ e 35,7 , respectivamente. Observou-se ainda cm 1990 que os serviços que realizaram triagcm através de única reação sorológica foram aqueles que possuiam bancos de sangue próprios e estavam concentrados nos menores municípios (Tabcla 7). Pode-se constatar ainda que 92,2\% dos scrviços no ano de 1990 utilizaram-se de doadores voluntários, $73,4 \%$ de reposição, $40,6 \%$ de prédepósito e $34,4 \%$ de auto-transfusão, além de em geral utilizarem-se de doadores de diferentes categorias. Não foi detectada em nenhum serviço a categoria de doador remunerado. Quanto a utilização de sangue e componentes, vcrificou-se que $4,7 \%$ dos serviços utilizaram apenas sangue total, destacando-se o uso de derivados do sangue pela grande maioria dos serviços. Não foi constatado 0 
emprego de violeta de genciana para profilaxia da docnça de Chagas transfusional $\mathrm{cm}$ ncnhum dos dois inquéritos realizados.

\section{Discussão}

As informações analisadas, considerando-se a abrangência da amostra que incluiu $10 \%$ dos municípios do Estado de São Paulo, apontam para uma sensível melhoria das condições do controle do sangue. A análise dos dados coletados, associada ao inquérito de consistência realizado por sorteio $\mathrm{cm} \mathrm{10 \%} \mathrm{dos} \mathrm{municípios} \mathrm{inventariados,} \mathrm{mostra}$ que as iniormações colhidas espelham a realidade do problema uma vez que pudemos contar, nesta fase do trabalho, com a participação de técnicos da árca de vigilância sanitária.

Considerando-se a tomada de iniciativas legais para o controle de sanguc e derivados no final dos anos 80 e a Portaria 721 de 9.8 .89 , que aprova normas técnicas neste sentido, e cria Centros Regionais de Referência em Hemocentros Estaduais, que deveriam estar se responsabilizando pela capacitação de recursos humanos, era de se esperar uma alteração do quadro da hemoterapia $\mathrm{em}$ nível nacional e do Estado de São Paulo, particularizado no presente estudo, fato este que conseguimos apreender $\mathrm{cm}$ nosso material, pela maior participação de serviços que são atendidos pelos Hemocentros, quando comparada a situação de 1990 (aumento de 8,5\% para 13,6\%. Deve-se levar $\mathrm{cm}$ conta que o númcro de Scrviços Hemotcrípicos oficiais ampliou de 27, $\mathrm{cm} \mathrm{1988,} \mathrm{para} 170 \mathrm{~cm}$ jutho de 1992, sendo São Paulo o Estado onde o sistema mais expandiu neste periodo'.

Embora a participação dos Hemocentros tenha sido constatada apenas nos municípios com mais do que 50.000 habitantes, como resultado de cxpansão das atividades do Programa Oficial de Sangue c Hemoderivados, a cobertura das pequenas cidades pela rede de Hemocentros é uma cxpectaliva que se avizinha a curto prazo. Dados cstimativos fornecidos pela COSAH mostram que no ano de 1991, 30,0\% da hemotcrapia praticada no Brasil foi realizada pela rede oficial (Hcmoccn(ros), sendo que no Estado de São Paulo esta rede foi responsável por $48,9 \%$ das transfusõcs rcalizadas*. Tais dados confirmam a rípida c progrcssiva ampliação do Sistema no Estado, como resultado de uma decisão política da Secretaria de Saúde, implementada a partir de 1988.

Quanto às caractcrísticas dos doadorcs, obscrvou-se que a maioria dos serviços $(92,2 \%)$ utiliza o

\footnotetext{
* Comunicação Pessoal.
}

doador voluntário. Este dado não permite apontar o pcrcentual de voluntários entre o total de doadores, uma vez que cada serviço refere a utilização de mais de um tipo de doador. No entanto, observou-se ausência total de doador remunerado, o que revela melhoria e amadurecimento do sistema ${ }^{10}$. $O$ encontro de $4,7 \%$ dos serviços que utilizam apenas sangue total, serviços estes localizados em municípios pequenos e, a existência de estrutura diferenciada para o fracionamento do sangue, principalmente nos municípios com mais de 50.000 habitantes, aponta para uma correlação positiva entre extrato populacional e qualidade dos serviços prestados.

Com relação ao número de transfusões, doadores e sorologia para doença de Chagas, observamos que a proporção transfusão/doadores foi respectivamente 1,05 e 1,32 , em 1988 e 1990 , mostrando melhoria no aproveitamento do sangue coletado. No entanto, ao se calcular o cocficiente de transfusão nos municípios trabalhados, obscrvou-se que 0 mesmo variou de 17,4 transfusões $/ 1.000$ habitantes $\mathrm{cm} 88$ para 23,7 transfusões $/ 1.000$ habitantes $\mathrm{em}$ 90. Tambćm se observou um incremento de $39,2 \%$ na proporção transfusões/serviço, que passou de 762, em 1988, para 1.011, em 1990. Assim, se por um lado tenha sido possível apreender no material estudado melhoria no aproveitamento do sangue, por outro lado, o aumento das indicações de transfusão observado aponta no sentido da real necessidade de uma triagem rigorosa do sangue a ser transfundido, de modo a se evitar novos casos de docnças adquiridas por essa via. O encontro de prevalência sorológica para $T$. cruzi próximo de $1,2 \%$ evidencia sensível redução da infecção chagásica nos úllimos anos, quando comparada aos dados apresentados em recentes revisões 6 ,

A análise da triagem laboratorial dos doadores aponta para um número rapidamente crescente de serviços que a realiza de forma adequada ${ }^{10}$, como previsto na legislação $\mathrm{cm}$ vigor. No entanto, nenhuma das doenças transmissíveis pelo sangue alcançou índice de triagem sorológica de $100 \%$ nos dois levantamentos realizados. Tomando apenas a infecção chagásica como exemplo, observamos que $94,2 \%$ dos serviços realizaram triagem $\mathrm{cm}$ 1990 contra os 69,0\% referidos $\mathrm{cm} 1988$. Ao lado deste fato, coloca-se a incorporação de técnicas mais sensívcis nas rotinas dos laboratórios, justificadas por $60,2 \%$ de scrviços que estão se utilizando da lécnica de ELISA, e o abandono daquelas de menor sensibilidade como a reação de fixação de complemento que era utilizada $\mathrm{cm} 1988$ por $28,6 \%$ dos scrviços e em 1990 por apenas 3,1\%. Outro aspecto importante a ser considerado é a maior participação de serviços que realizam dois ou mais testes para triagem da doença de Chagas, totalizando $92,8 \%$. 
$\mathrm{Na}$ atual fase do controle da doença de Chagas no Estado de São Paulo, quando já não se apreende mais a transmissão natural através de triatomíneos domiciliados ${ }^{7,8}$, coloca-se em evidência mecanismos alternativos de transmissão da tripanossomíase, dos quais a transfusão de sangue tem sido considerada a mais importante.

O presente inquérito sobre o controle de hemoterapia fornece elementos úteis para avaliação do risco de transmissão da endemia chagásica através do sangue, cujo controle definitivo no Estado de São Paulo pode-se antever a curto prazo, considerando-se a política de melhoria da qualidade do sangue transfundido, implementada pelas autoridades de saúde do Estado.

WANDERLEY, D.M.V. et al. [Inquiry into the control of hemotherapy and transfusional Chagas' disease: 1988 and 1990]. Rev. Saúde Pública, 27: 430-5, 1993. An inquiry with the objective of bringing the study of the hemotherapy situation in the state of S. Paulo, up-to-date was undertaken in 1990 and compared with that carried out in 1988. In 1990 research was undertaken in 62 counties, 48 of which performed blood transfusions through 104 services, with hemocenter participation in $13.5 \%$ of these latter. In 1988 the respective figures were $57,40,71$ and $8.5 \%$. The number of donors and transfusions and prevalence of serological positivity for Chagas' disease were, respectively: 105,170; 79,544 and $1.24 \%$, while in 1988 the corresponding numbers were: 51,$614 ; 49,211$ and $1.52 \%$. Evaluating the serological selection it was found that the following percentages of the services performed serological tests: for Chagas' disease $(94.2 \%)$, siphylis $(94.2 \%)$, hepatitis $(94.2 \%)$, HIIV (92.3\%) and malaria (20.2\%) while in 1988 only $69.0 \%$; $70.4 \% ; 67.6 \%, 63.4 \%$ and $16.9 \%$, respectively, of the services utilized these same tests. In respect of the serological trial specific for Chagas' disease the results were: complement fixation- $4.9 \%$; latex - $4.0 \%$; indirect immunofluoresce - $80 \%$; direct hemaglutination $24.0 \%$, indirect hemaglutination - $69.0 \%$ and ELISA $59.0 \%$ of all services. In the preceding inquiry only $14.3 \%$ of the services performed the ELISA test, and complement fixation was utilized in $28.6 \%$. The results show that the services are utilizing ever more sensitive techniques in the serological routine. Further it was observed that in $1988,77.6 \%$ of all the services studied used only one tocnique for the diagnosis of Chagas' disease while in $199092.9 \%$ utilized two or more different tecniques. It is concluded that the quality of the hemotherapy undertaken in the state of $\mathrm{S}$. Paulo has improved significantly.

Keywords: Blood banks, statistical. Trypanosomiasis South-American, transmission. Serodiagnosis, statistical.

\section{Referências Bibliográficas}

1. COSTA, E. \& SIIVA, M. Relatório das atividades da Coordenação Nacional de Sangue e Hemoderivados do Ministério da Saúde. Brasília, 1992. [mimeografado].

2. GONTJUO, E.C.D. Doença de Chagas transfusional na regiâo metropolitana de Belo Horizonte: aspectos clínico-epidemiológicos e a questão institucional. Belo Horizonte. 1989. [Tese de Doutorado - Universidade Federal de Minas Gerais].

3. MAZZA, S. et al. Transmission del Schistosoma cruzi al niño por leche de la madre con enfermedad de Chagas. Mis. Est. Pat. Reg. Arq., 28: 41, 1936.

4. MORAES-SOUZA, H.; WANDERLEY, D.M.V.; BRENER, S.; NASCIMENTO, R.D.; ANTUNES, L.M.; DIAS, J.C.P. Hemoterapia e doença de Chagas transfusional no Brasil: resultados de um inquérito nacional. Bol. Of. Sanit. Panamer. [no prelo].

5. PELLEGRNNO, J. O perigo da transmissão da doença de Chagas pela transfusâo de sangue: primeiras comparaçôes sorologicas de esquizotripanose em doadores e em candidatos a doadores de sangue. Brasil Med., 63: 65-71, 1949.

6. SCHIMUNIS, G.A. Trypanosoma cruzi, the ethiologic agent of Chagas' discase: status in the blood supply in endemic and nonendemic countries. Transfusion, 31: 547-57, 1991.

7. WANDERLEY, D.M.V. Análise da atual estratégia do Programa de Controle da doença de Chagas no Estado de São Paulo. São Paulo, 1987. [Dissertação de Mestrado Faculdade de Saúde Pública da USP].

8. WANDERLEY, D.M.V. Vigilância entomológica da doença de Chagas no Estado de São Paulo. Rev. Saúde Pública, 25: 28-32, 1991.

9. WANDEL, S. \& DIAS, J.C.P. Transfusion transmitted Chagas' disease. In: Wendel, S.; Brener, Z; Camargo, M.E.; Rossi, A. Chagas disease (American Trypanosomiasis): its impact on transfusion and clinical medicine. São Paulo, ISBT Brazil' 92, 1992. p. 103-33.

10. WORLD HEALTII ORGANZATION EXPERT COMMITTEE ON TIE CONTROL OF CHAGAS DISEASE, Bucnos Aires, 1989. Report. Geneva, 1991. (WIIO Tecnical Report Series, 811).

Recebido para publicaçäo em 18.5 .93 Reapresentado em 22.9.1993 Aprovado para publicação em 27.10.1993 